\title{
On the Performance of Secure Vehicular Communication Systems
}

\author{
Giorgio Calandriello*, Panos Papadimitratos ${ }^{\dagger}$, Jean-Pierre Hubaux ${ }^{\dagger}$, Antonio Lioy* \\ ${ }^{*}$ DAUIN, Politecnico di Torino, Italy \\ \{giorgio.calandriello, antonio.lioy\}@polito.it \\ ${ }^{\dagger}$ LCA, IC, EPFL, Switzerland \\ \{panos.papadimitratos, jean-pierre.hubaux\}@epfl.ch
}

\begin{abstract}
Vehicular communication (VC) systems are being developed primarily to enhance transportation safety and efficiency. Vehicle-to-vehicle communication, in particular, frequent cooperative awareness messages or safety beacons, has been considered over the past years as a main approach. Meanwhile, the need to provide security and to safeguard users' privacy is well understood, and security architectures for VC systems have been proposed. Although technical approaches to secure VC have several commonalities and a consensus has formed, there are critical questions that have remained largely unanswered: Are the proposed security and privacy schemes practical? Can the secured VC systems support the VC-enabled applications as effectively as unsecured VC would? How should security be designed so that its integration into a VC system has a limited effect on the system's performance? In this paper, we provide answers to these questions, investigating the joint effect of a set of system parameters and components. We consider the state-ofthe-art approach in secure $\mathrm{VC}$, and we evaluate analytically and through simulations the interdependencies among components and system characteristics. Overall, we identify key design choices for the deployment of efficient, effective, and secure VC systems.
\end{abstract}

Index Terms-Security and Protection, Wireless communication, Reliability, Availability, and Serviceability

\section{INTRODUCTION}

Vehicular communication (VC) systems will comprise vehicles and fixed road-side equipment (RSU) with wireless transceivers, and sensing and processing units. Vehicle-tovehicle (V2V) and vehicle-to-infrastructure (V2I) communication will enable a range of applications, with transportation safety playing a predominant role. Almost all research and development efforts converge to safety applications based on $\mathrm{V} 2 \mathrm{~V}$ communication, with vehicles frequently beaconing their status (e.g., position, speed, direction), along with warnings about potential dangers.

Nonetheless, VC systems can be vulnerable to attacks and jeopardize users' privacy: An adversary could, for example, inject beacons with false information, or collect vehicles' messages to track their locations and infer sensitive user data. Industry, academia, and authorities have recently understood that security and privacy protection are prerequisites for the deployment of VC systems. Security architectures were developed by the IEEE 1609.2 working group [1], the SeVeCom project [2], [3], following the earlier NoW project [4] and now the Car-to-Car Communication Consortium (C2C-CC) [5] and the eSafety eSecurity WG activities [6].
Across projects and working groups, secure $\mathrm{VC}$ systems rely on public key cryptography and digital signatures to protect V2V and V2I messages; Certification Authorities (CAs) manage credentials for legitimate participants (vehicles and RSUs). Pseudonymous authentication, with vehicles using short-lived credentials and public-private key pairs, provides protection of privacy along with security (authentication, integrity and nonrepudiation as primary requirements). Security mechanisms protect all traffic sent across the $802.11 \mathrm{p}$ data link [7], including the safety beacons each vehicle transmits, typically every 100 to $300 \mathrm{~ms}$.

Adding security for this high-rate communication would incur high overhead, both in terms of communication and processing. Consider, for example, a vehicle receiving digitally signed safety beacons from a hundred vehicles within range; it would need to validate a high percentage or almost all of those within a short delay in the order of a hundred milliseconds [7]. Even if VC is effective under such dense network conditions, the additional security overhead could cause failure in meeting the delay and reliability requirements of safety applications. This is especially so because the VC environment lacks abundant resources (bandwidth, computational power).

The following question naturally follows: Can secure VC systems be practical? Given the current system constraints and design approaches, could the addition of security and privacy mechanisms make VC systems ineffective? We address this problem in this paper, building on our previous work ( [8], [9]). Based on broadly accepted approaches for secure and privacyenhancing VC [1]-[4], we first outline how pseudonymous authentication is possible without repeated interactions with the CAs. Then, we present a proposal for reducing the security overhead without harming the effectiveness of the VC system, and we investigate how variants of secure VC instantiations affect the system performance. In particular, our main contribution is the first comprehensive evaluation of secure VC operations: (i) We evaluate the communication reliability, and then (ii) we determine if and how VC nodes can sustain the incurred processing load, providing an approximate analytical evaluation and closely matching simulation results. Having determined if VC nodes have sufficient processing power, (iii) we consider the overall system performance with respect to transportation safety and (iv) transportation efficiency, evaluating secure VC-enabled applications for a broad range of system configurations. We find that appropriately designed 
security and privacy-enhancing VC systems can essentially support a safety application as effectively as unsecured VC systems can. Moreover, (v) we investigate revocation and propose a practical method for anonymous authentication schemes in $\mathrm{VC}$, and (vi) we discuss additional technical issues and demonstrate how our model can assess processing resources needed for future systems.

We discuss the problem at hand and outline our investigation approach in Sec. II and present the representative secure and privacy-enhancing VC schemes we evaluate in Sec. III. The simulation setup, our analysis and experimental results follow in Sec. IV-Sec.VIII, along with the treatment of revocation in Sec. IX and other technical issues and extensions for future instantiations in Sec. X. We discuss related work in Sec. XI before we conclude.

\section{Problem AND APPROACH OVERVIEW}

We want to determine whether the broadly accepted state of the art of secure VC is viable, especially considering how challenging VC environments are; because heavy-traffic scenarios (thus, dense network topologies) - with tens, one hundred or more vehicles (nodes) within range - can often occur. The traditional approach has been to analyze the protocol overhead and the network performance. However, in VC systems the objective is not to have a well-performing network per se, but to effectively support VC-specific applications. This is why we investigate the overall system performance, considering five dimensions: (i) communication technology, (ii) system resources, (iii) network configuration and environmental factors, (iv) security protocols, and (v) supported applications.

The technology commonly accepted for V2V and V2I communication is the IEEE 802.11p [10], which is incorporated in the Dedicated Short Range Communication (DSRC) - Wireless Access in a Vehicular Environment (WAVE) [11] and the Communication Access for Land Mobiles (CALM) [12] standards. Vehicles transmit periodic safety beacons on one dedicated channel, at a system-selectable beaconing rate. Bandwidth, one of the primary system resources, is determined by the standards, and it is considered fixed for this investigation. The second primary resource, processing power, can be adapted. Here, we take into consideration platforms that are currently used in VC prototypes, but any system should have sufficient processing power for its designated tasks. Thus, the system designer can always increase the processing power at the expense of increased cost.

The use of specific cryptographic primitives and other protocol functionalities determine the processing load for each node (vehicle). Here we consider the basic pseudonymous authentication approach, which has gained broad acceptance: It provides message authentication, integrity, non-repudiation and it makes it hard for two or more messages from the same sender to be linked ${ }^{1}$. Given the large number of temporary identities (pseudonyms) in the system, pseudonymous

\footnotetext{
${ }^{1}$ More precisely, it allows that messages produced by a node over a protocol-selectable period of time, $\tau$, be linked. But messages $m_{1}, m_{2}$ generated at times $t_{1}, t_{2}$ respectively, such that $t_{2}>t_{1}+\tau$, should not be linkable. The shorter $\tau$ is the fewer the linkable messages are and the harder tracking a node becomes.
}

authentication can become cumbersome to manage. Therefore, we consider here a novel scheme, first presented in [8], [9], to alleviate this constraint, thanks to a more powerful but also more expensive anonymous authentication primitive. We describe these security protocols in Sec. III.

We consider transportation safety and efficiency applications as they are distinctive features of VC systems (compared to other mobile computing systems) and two main driving forces for the VC systems deployment. Moreover, they are, especially the safety ones, the most challenging among $\mathrm{VC}$ enabled applications; their stringent time constraints and their critical nature can affect the well-being of the vehicle passengers. We focus here on one safety application, Emergency Braking Notification (EBN), and one efficiency application, Decentralized Floating Car Data (DFCD).

In order that the appropriate processing power can be determined and provisioned, we provide a framework to analyze the effect of a given processing load on the node performance. Then, we consider a system for which processing is not a bottleneck (otherwise, the system would certainly fail) and we evaluate the effectiveness of the applications. Conversely, given appropriate design choices (i.e., equipment with sufficient power), our investigation reveals the effect of other parameters and their interdependencies.

\section{SECURE COMMUNiCATION}

Each node (vehicle) has a long-term, unique identity and corresponding credentials managed by a Certification Authority (CA); without loss of generality, we assume there is a single $\mathrm{CA}$, even though in reality a CA hierarchy would be present [13]. Instead of using their long-term credentials, vehicles obtain from the CA and use a set of short-lived certified public keys that do not identify the vehicle; then, they digitally sign messages with the corresponding private keys. As this is the widely used approach of pseudonymous authentication [1][5], we refer to it as the Baseline Pseudonym (BP) scheme, and define its operation in Sec. III-A. We consider only the vehicles, as the privacy of RSUs or other infrastructure does not need to be protected.

As the BP scheme requires numerous short-lived certificates and keys per vehicle, the stronger the protection of privacy is sought the higher the number of identities would be. For large-scale systems, this and the cost of periodically preloading vehicles with temporary keys and credentials can become a significant burden. To reduce the key management complexity and enhance the system usability and efficiency, we propose that nodes self-generate, i.e., self-certify, their own pseudonyms. With this method, first described in [8], [9], vehicles do not need to be side-lined or to compromise their user's privacy if a "fresh" pseudonym is no longer available; no "over-provisioning" in the supply of pseudonyms is necessary; and the cost of obtaining new pseudonyms over an "out-ofband" channel is avoided.

This can be achieved with the use of anonymous authentication primitives, notably Group Signatures $(G S)$ we describe in Sec. III-B. As GS is hard to use for all VC messages, because of the GS processing and communication overhead, 
in Sec. III-C we propose our Hybrid Pseudonym (HP) scheme that allows vehicles to generate on-the-fly their pseudonyms, by combining the BP and GS approaches. HP alleviates the management overhead of the BP, but in principle it is more costly than BP (due to HP's use of GS). To reduce the cost of HP to equal roughly that of BP and to increase the robustness of any pseudonymous approach, we propose a set of optimizations (Sec. III-D).

Concerning revocation (Sec. IX and X), all the approaches make use of Revocation Lists (RL), generated by the CA and distributed to vehicles primarily via the infrastructure [2], [13]. When a node validates a certificate, it checks whether the sender is revoked; if successful (i.e. the sender is not revoked), it proceeds with validating the message (signature(s)).

\section{A. Baseline Pseudonym (BP) Scheme}

Each node $V$ is equipped with a set of pseudonyms that are certified public keys without any information that identifies $V$. More specifically, for the $i$-th pseudonym $K_{V}^{i}$ for node $V$, the CA provides a certificate $\operatorname{Cert}_{C A}\left(K_{V}^{i}\right)$, which is simply a CA signature on the public key $K_{V}^{i}$ (unlike the common notion of certificate, for example the X.509 certificate). The node uses the private key $k_{V}^{i}$ for the pseudonym $K_{V}^{i}$ to digitally sign messages. To enable message validation, the pseudonym and the certificate of the signer are attached in each message. With $\sigma_{k_{V}^{i}}($ ) denoting $V$ 's signature under its $i$-th pseudonym and $m$ the signed message payload, the message format is:

$$
M 1: m, \sigma_{k_{V}^{i}}(m), K_{V}^{i}, \operatorname{Cert}_{C A}\left(K_{V}^{i}\right)
$$

Upon receipt of $M 1$, a node, with the public key of the CA available, validates $\operatorname{Cert}_{C A}\left(K_{V}^{i}\right)$, and then verifies the signature using $K_{V}^{i}$.

Each pseudonym is used at most for a period $\tau$ (referenced in the rest of the paper as the pseudonym lifetime) and then discarded. We abstract away a number of possible implementation aspects, such as (i) the dynamic adaptation of the period of pseudonym usage, (ii) the number of pseudonyms $\left(K_{V}^{i}\right.$ and the corresponding $k_{V}^{i}, \operatorname{Cert}_{C A}\left(K_{V}^{i}\right)$ ) that are preloaded to $V$, (iii) the frequency of pseudonym refills, and (iv) policies for pseudonym change, such as factors rendering a pseudonym change unnecessary (e.g., a TCP connection to an access point), and interactions of pseudonym changes with the network stack [14]. All these are important yet largely orthogonal to this investigation. The CA maintains a map from the long-term identity of $V$ to the $\left\{K_{V}^{i}\right\}$ set of pseudonyms provided to a node. If presented with a message $M 1$, the CA can perform the inverse mapping and identify the signer.

\section{B. Group Signature (GS) Scheme}

Each node $V$ is equipped with a secret group signing key $g s k_{V}$, with the group members comprising all vehicles registered with the CA. A group public key $G P K_{C A}$ allows for the validation (by any node) of any group signature $\Sigma_{C A, V}$ generated by a group member. Intuitively, a group signature scheme allows any node $V$ to sign a message on behalf of the group, without $V$ 's identity being revealed to the signature verifier. Moreover, it is impossible to link any two signatures of a legitimate group member. Note that no public key or other credentials need to be attached to an anonymously authenticated message; the format is:

$$
M 2: m, \Sigma_{C A, V}(m)
$$

Group signatures, introduced by Chaum [15], are revisited in numerous works, e.g., [16]-[19], with formal definitions in [20], [21]. For the rest of the discussion, we assume and use the group signature scheme proposed in [22]. If the identification of a signer is necessary, the CA can perform an Open operation [20], [21] and reveal the signer's identity.

\section{Hybrid Pseudonym (HP) Scheme}

The combination of the BP and GS schemes is the basic element of our proposal [8], [9]. Each node $V$ is equipped with a group signing key $g s k_{V}$ and the group public key $G P K_{C A}$ (recall that the group is the total of vehicles registered with the CA). Rather than generating group signatures to protect messages, a node generates its own set of pseudonyms $\left\{K_{V}^{i}\right\}$ (according to the BP public key cryptosystem). As for the BP scheme (Sec. III-A), a pseudonym is a public key without identification information, and $\left\{k_{V}^{i}\right\}$ is the set of corresponding private keys. For HP, the CA does not provide a certificate on $K_{V}^{i}$; instead, $V$ uses $g s k_{V}$ to generate a group signature $\Sigma_{C A, V}()$ on each pseudonym $K_{V}^{i}$ instead. In other words, it generates and "self-certifies" $K_{V}^{i}$ on-the-fly, by producing $\Sigma_{C A, V}\left(K_{V}^{i}\right)$. Similarly to $M 1, V$ attaches $\Sigma_{C A, V}\left(K_{V}^{i}\right)$ to each message, and signs with the corresponding $k_{V}^{i}$ :

$$
M 3: m, \sigma_{k_{V}^{i}}(m), K_{V}^{i}, \Sigma_{C A, V}\left(K_{V}^{i}\right)
$$

When a node receives a message $M 3$, the group signature $\Sigma_{C A, V}\left(K_{V}^{i}\right)$ is verified, using $G P K_{C A}$. If successful, the receiver infers that a legitimate system (group) member generated pseudonym $K_{V}^{i}$. We emphasize that, as per the properties of group signatures, the receiver/verifier of the certificate cannot identify $V$ and cannot link this certificate and pseudonym to any prior pseudonym used by $V$. Once the legitimacy of the pseudonym is established, the validation of $\sigma_{k_{V}^{i}}(m)$ is identical to that for $M 1$. To identify the message signer, an Open on the $\Sigma_{C A, V}\left(K_{V}^{i}\right)$ group signature is necessary; message $m$ is bound to $K_{V}^{i}$ via $\sigma_{k_{V}^{i}}(m)$, and $K_{V}^{i}$ is bound to $V$ via $\Sigma_{C A, V}\left(K_{V}^{i}\right)$. Fig. 1(a) compares the BP and HP.

\section{Optimizations for the BP and HP Schemes}

We describe optimizations to reduce overhead (Optimizations 1 and 2) and enhance robustness (Optimization 3). We employ the notation of the HP scheme, but the same considerations hold for BP too. Fig. 1(b) summarizes Optimizations 2 and 3 .

Optimization 1: On the sender's side, $\Sigma_{C A, V}\left(K_{V}^{i}\right)$ is computed only once per $K_{V}^{i}$, because $\Sigma_{C A, V}\left(K_{V}^{i}\right)$ remains unchanged throughout the pseudonym lifetime $\tau$. For the same reason, on the verifier's side the $\Sigma_{C A, V}\left(K_{V}^{i}\right)$ is validated upon the first reception and stored, even though the sender appends it to multiple (all) messages. For all subsequent receptions, if $\Sigma_{C A, V}\left(K_{V}^{i}\right)$ has already been seen, the verifier skips its validation. This optimization is useful because in practice $\tau \gg$ $\gamma^{-1}$, where $\gamma$ is defined as the beacon frequency. 


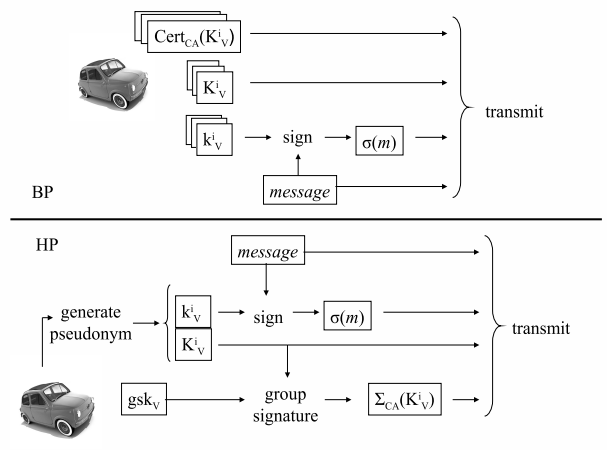

(a) Illustration of the BP and HP schemes.
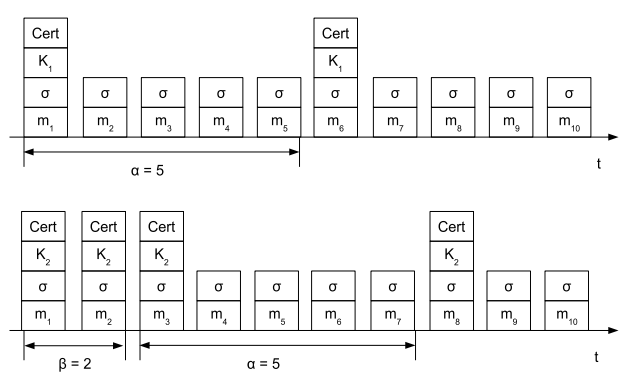

(b) Illustration of Optimizations 2 and 3, with $\alpha=5$ and $\beta=2$. One $L O N G$ message is sent every 5 , and repeated 2 times after a pseudonym renewal.

Fig. 1: Illustration of the BP and HP security schemes and related optimizations.

Optimization 2: The sender appends its signature $\sigma_{k_{V}^{i}}(m)$ to all messages, but it appends the corresponding $K_{V}^{i}, \Sigma_{C A, V}\left(K_{V}^{i}\right)$ only once every $\alpha$ messages. We term such messages (M1 and M3) as LONG. M4 is defined as follows:

$$
M 4: m, \sigma_{k_{V}^{i}}(m)
$$

We denote M4 as SHORT, and $\alpha$ as the Certificate Period. $\alpha \in$ $[1, \tau \gamma]$, where $\tau \gamma$ is the total number of transmissions during the pseudonym lifetime $\tau$. To allow the user to choose the right $K_{V}^{i}$ to verify an incoming SHORT message, all messages will carry a randomly generated 4-byte keyID field. This does not affect privacy as all SHORT messages signed under the same $K_{V}^{i}$ can be trivially linked.

When a pseudonym change occurs, the new triplet $\sigma_{k_{V}^{i+1}}(m), K_{V}^{i+1}, \Sigma_{C A, V}\left(K_{V}^{i+1}\right)$ must be computed and transmitted. $V$ will sign messages with the new $k_{V}^{i+1}$ corresponding to $K_{V}^{i+1}$ from then on.

Optimization 2 can affect the protocol robustness, if the message that carries $K_{V}^{i+1}, \Sigma_{C A, V}\left(K_{V}^{i+1}\right)$ is not received. Then, nodes in range of $V$ must wait for $\alpha$ messages for the next pseudonym transmission, while being unable to validate any message from $V$. This can be dangerous if vehicles are close to each other and/or are moving at high relative speeds. Thus, we propose the following scheme to mitigate this problem.

Optimization 3: $V$ repeats the transmission of $K_{V}^{i+1}, \Sigma_{C A, V}\left(K_{V}^{i+1}\right)$ for $\beta$ consecutive messages when $K_{V}^{i+1}$ is issued, with $\beta$ denoted as the Push Counter. After the $\beta$ repetitions, with $\beta \in[0, \alpha-1]$, the normal sequence $1 L O N G$, $\alpha-1$ SHORT starts again.

\section{Evaluation Overview}

We analyze the system performance of secure $\mathrm{VC}$ along the dimensions presented in Sec. II. Given the complexity of the problem, we employ simulation as a primary tool of analysis and we provide analytical approximations. We evaluate the effectiveness of the EBN and the DFCD applications in a variety of setups, to gain insight into the role of each of

\begin{tabular}{|l|c|c|c|}
\hline Parameter & Symbol & Range & Unit \\
\hline Certificate Period & $\alpha$ & $1,5,10,15,30,50$ & messages \\
Push Period & $\beta$ & 0 to 10 & messages \\
Beacon Frequency & $\gamma$ & 3.33 and 10 & beacons/s \\
Pseudonym Lifetime & $\tau$ & 60 & $s$ \\
Number of Neighbors & $N$ & $160,240,320$ & vehicles \\
Packet Payload & $m$ & 200 & bytes \\
Initial Vehicle Spacing & $s$ & $20 \pm 1.5,150,200$ & $m$ \\
Average Vehicle Speed & $v$ & 65 and 80 & $\mathrm{Km} / \mathrm{h}$ \\
Road Setup & - & $4,6,8$ & lanes \\
Security Schemes & - & BP and HP & - \\
Nominal Communic. Range & $r$ & 200 & $m$ \\
\hline
\end{tabular}

TABLE I: System parameters and values assigned for the evaluation.

the system parameters; indicative values for these are summarized in Table I. We study challenging or even extreme transportation conditions, because the system has to remain operational even under these conditions. We are interested in parameters that are not related to security (and of course in security protocol parameters) precisely because we want to capture their importance for the secure VC system.

We assume that only vehicles transmit, because RSUs would always be less numerous (each serving an area with tens or hundreds of vehicles), and often completely absent; thus, almost all of the safety-related data will be generated by vehicles. Finally, we also assume that all beacons carry relevant information for safety applications. We couple the $n s-2$ simulator, which simulates $\mathrm{V} 2 \mathrm{~V}$ communication, with a custom module written in $\mathrm{C}$, which simulates (i) the EBN and DFCD applications and its effect on vehicles movement and (ii) the security processing of messages. We choose such a combination because we could not find another publicly available simulation environment with security functionality integrated and with nodes adjusting their behavior according to the messages they receive.

First, we evaluate the cryptographic overhead, in terms of communication and processing, and we choose representative cryptographic primitives, security levels, and a reference platform (Sec. V) and then analyze the communication reliability (Sec. VI). Based on these two elements, we study the effect 


\begin{tabular}{|c|c|c|c|c|c|c|}
\hline Algorithm & $\begin{array}{c}\text { Security level } \\
\text { (bits) }\end{array}$ & $\begin{array}{c}\text { Sign } \\
\text { (ms) }\end{array}$ & $\begin{array}{c}\text { Verify } \\
\text { (ms) }\end{array}$ & $\begin{array}{c}\text { Signature } \\
\text { (bytes) }\end{array}$ & $\begin{array}{c}\text { Public key } \\
\text { (bytes) }\end{array}$ & $\begin{array}{c}\text { Private key } \\
\text { (bytes) }\end{array}$ \\
\hline \hline ECDSA-192 & 96 & 0.5 & 3 & 48 & 25 & 24 \\
ECDSA-256 & 128 & 0.8 & 4.2 & 64 & 33 & 32 \\
GS & 128 & 53.7 & 49.3 & 225 & 800 & 64 \\
\hline
\end{tabular}

TABLE II: Computation costs on a $1.5 \mathrm{GHz}$ Centrino processor and communication overhead for different signing algorithms: Elliptic Curve Digital Signature Algorithm (ECDSA) using different standardized elliptic curves, and a representative efficient Group Signature (GS) algorithm [22].

\begin{tabular}{|c||c|c|c|}
\hline & Sign (ms) & Verify (ms) & Overhead (bytes) \\
\hline BP LONG & 1.3 & 7.2 & 141 \\
HP LONG & 54.2 & 52.3 & 302 \\
SHORT & 0.5 & 3 & 52 \\
\hline
\end{tabular}

TABLE III: Processing delay (in ms) and communication overhead (in bytes) for different packet types.

\begin{tabular}{|c|c|}
\hline & Packets per beacon period $\gamma^{-1}$ \\
\hline BP LONG & 13.9 \\
HP LONG & 1.9 \\
SHORT & 33.3 \\
\hline
\end{tabular}

TABLE IV: Maximum number of verifiable packets per $\gamma^{-1}$ s, for $\gamma=10$.

of the processing overhead on individual nodes (Sec. VII) and develop an approximate model. Assuming that nodes are provisioned with sufficient processing power, we perform a system-wide analysis for the considered applications (Sec. VIII). Finally, we address revocation, which can be costly (Sec. IX), and demonstrate how processing power provisioning can be done for future secure VC systems (Sec. X).

\section{CRyptographic Overhead}

We use EC-DSA as the basic signature algorithm [23], the group signature algorithm proposed by [22], and security level of $t=96$ bits for message signatures and $t=128$ bits for CA certificates in BP and for group signatures used in GS and HP. High security might not be necessary for the short-lived $K_{V}^{i}$, but it is required for the long-term keys and CA certificates.

Overhead: The $K_{V}^{i}, \operatorname{Cert}_{C A}\left(K_{V}^{i}\right)$ is 89 bytes for BP, and with $\sigma_{k_{V}^{i}}(m)$ and KeyID the overhead is 141 bytes per message. For GS, the overhead is $\Sigma_{C A, V}(m)$, thus 225 bytes per message. For HP, the overhead is $\sigma_{k_{V}^{i}}(m), K_{V}^{i}, \Sigma_{C A, V}\left(K_{V}^{i}\right)$, KeyID, in total 302 bytes per message. For the $\alpha-1$ SHORT messages, the overhead is $\sigma_{k_{V}^{i}}(m), K e y I D$, thus 52 bytes. The effective overhead reduction depends on the value of $\alpha$ (see Sec. VI).

Computation: We make use of a Centrino machine with the clock speed set at $1.5 \mathrm{GHz}$, which is close to the CVIS (Cooperative Vehicle-Infrastructure System) vehicle PC, a rather powerful platform (compared to generally available embedded processors) adopted for the development of future VANET applications [24]. We obtain an EC-DSA benchmark on the platform through the OpenSSL standard test suite [25]. As for group signatures, a well-established implementation of the chosen algorithm [22] is not yet available. Thus, to estimate the processing delay, we calculate the number of 32bit word scalar multiplications required for GS signing and verifying; we extract the relevant data from [26] and [27] and we benchmark the scalar multiplication operation.

Table II shows the costs for signature, verification and overhead for the chosen algorithms. To obtain individual processing delays for a given type of message, it suffices to take the sum of the corresponding cryptographic primitive delays (M1, M3 and M4). Security levels are $t=96$ for $\sigma_{k_{V}^{i}}(m)$, and $t=128$ for $\operatorname{Cert}_{C A}\left(K_{V}^{i}\right), \Sigma_{C A, V}(m)$ and thus $\Sigma_{C A, V}\left(K_{V}^{i}\right)$; we summarize results per message in Table III.

\section{Communication Reliability}

The communication reliability is of central importance and depends on the channel properties and load; the more loaded the channel is, the more likely it is for a packet collision to occur at the wireless medium, which depends on the number of transmitters, $N$, the beacon frequency, $\gamma$, and the packet size (including the security overhead). We implement beacons with information on vehicle position, speed, and direction, with a timestamp, and safety warnings in a payload, $m$, of 200 bytes. The physical layer models realistic radio propagation [28], [29], with a nominal communication range of $r=200 \mathrm{~m}$ and a bandwidth of $6 \mathrm{Mb} / \mathrm{s} \mathrm{[7],} \mathrm{[30],} \mathrm{[31].}$

We estimate, with the help of detailed simulations, the average probability of successful reception at a receiving node at the center of a 200-meter radius disc that covers the entire width of a multi-lane highway and is filled with $N$ uniformly spaced neighbors. We consider various settings, increasing the number of lanes and decreasing the vehicle density, varying the size of $N$ from 8 to 160; a subset of these settings (four-, sixand eight- lanes with average spacing of 20 meters) is used in Sec. VIII. This metric is independent of the distance between the transmitter and the receiver. The reception probability as a function of the sender-receiver distance is presented in our previous work [9], and it is omitted here for simplicity and due to lack of space. The results are shown in Fig. 2 with 95\% confidence intervals and are repeated 5 times, with each repetition lasting $60 \mathrm{~s}$ of simulated time.

We note that the $802.11 \mathrm{p}$ broadcast communication, a CSMA/CA protocol without acknowledgements, could be modeled and evaluated analytically, for example, in terms of the probability of successful reception and throughput, following numerous works for similar CSMA/CA protocols under various assumptions (on traffic conditions, presence or absence or channel errors, packet sizes, impact of hidden terminals, etc). An effort to derive a precise analytical model for $\mathrm{VC}$ is orthogonal to our investigation. What we need here 


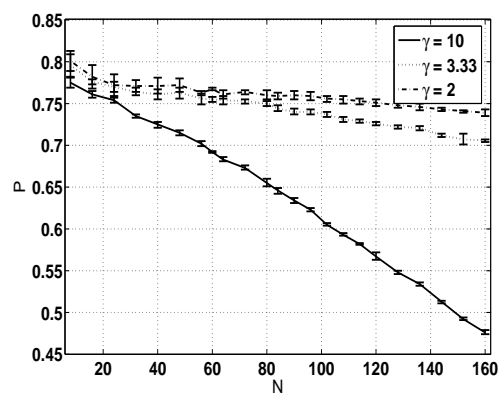

(a) Ratio of messages received for the HP scheme; $\alpha=10$.

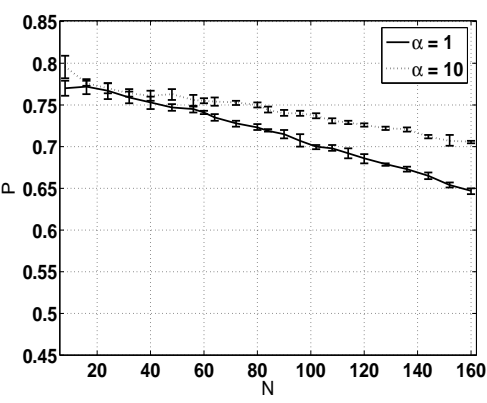

(b) Ratio of messages received for the $\mathrm{HP}$ scheme; $\gamma=3.33$ beacons/s.

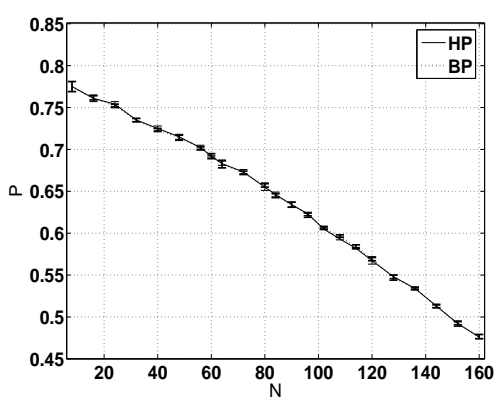

(c) Ratio of messages received for the $\mathrm{BP}$ and HP schemes; $\gamma=10$ beacons/s and $\alpha=10$.

Fig. 2: Reliability of message reception for secure VC (beaconing), as a function of the neighborhood size, $N$.

\begin{tabular}{|c|c|c|c|c|}
\hline $\begin{array}{c}\gamma \\
\text { (bcn./s.) }\end{array}$ & $\begin{array}{c}\mathbf{H P} \\
\alpha=1\end{array}$ & $\begin{array}{c}\mathbf{H P} \\
\alpha=10\end{array}$ & $\begin{array}{c}\mathbf{B P} \\
\alpha=1\end{array}$ & $\begin{array}{c}\mathbf{B P} \\
\alpha=10\end{array}$ \\
\hline 10 & $\overline{5020}$ & 27 & $\overline{3410}$ & 2609 \\
\hline 3.33 & 1671.66 & 922.41 & 1135.53 & 868.70 \\
\hline 2 & 1004 & 554 & 682 & 521.8 \\
\hline
\end{tabular}

TABLE V: Offered load per transmitter, in bytes/s, for different security schemes and settings.

is an accurate evaluation of the communication reliability, as a stepping stone for our security-related investigation; this can be obtained via detailed simulations.

With the fixed available bandwidth, specific for the communication technology, the communication reliability depends on the offered load; Table V summarizes the load for each of the scenarios in this paper. Fig. 2 shows the estimate of the probability of reception, $P$ (i.e. the ratio of received messages over transmitted beacons), as a function of the number of transmitters, $N$, the beacon frequency, $\gamma$, and the protocol parameter $\alpha$. We observe that the communication performance degrades fast with $N$ when $\gamma$ is high (Fig. 2(a) for HP), whereas the degradation is much slower as $N$ increases for lower $\gamma$ values. The effect of increasing $\alpha$, thus reducing overhead is significant even when $\gamma$ is not very high (e.g., $\gamma=3.33$ beacons/s, Fig. 2(b) again for HP). Finally, as shown by Fig. 2(c) for $\gamma=10$ and $\alpha=10$, the BP and HP schemes perform almost identically.

These results show that $\gamma$ turns out to be the most significant channel load factor. Choosing a smaller value for $\gamma$ decreases the channel saturation and thus the processing overhead (fewer messages are sent); but it also affects the transportation safety, as we show in Sec. VIII. At the same time, the appropriate choice of BP and HP parameters can reduce security overhead (notably $\alpha$, as it will become clear in Sec. VIII the needed $\beta$ values incur very limited overhead). The almost identical $P$ for BP and HP also show the benefit from the proposed optimizations, as both schemes have comparable overhead (with the advantages of HP).

\section{Processing Overhead}

We want to answer the following questions: (i) How many packets does a given node $V_{\mathcal{R}}$ have to verify per time unit, in various VC settings? (ii) What is the additional message verification delay introduced by security? We consider one beacon period, i.e. $\gamma^{-1}$ seconds, as the time unit, as specified by transportation safety requirements.

The BP and HP schemes use two general message types, according to the induced security communication overhead: SHORT messages carrying a node signature, and $L O N G$ messages carrying a node signature and certificate. Each node transmits one LONG message every a SHORT messages, with $\beta$ additional consecutive $L O N G$ messages sent upon a pseudonym change.

The processing load at some node $V_{\mathcal{R}}$ depends on the number of packets it needs to verify. This consists primarily of signature verifications for essentially all received beacons, as they carry safety-related information. In a given slot, if $V_{\mathcal{R}}$ has $N$ neighbors in range, it should validate $O(N)$ messages per time unit. Due to Optimization $1, V_{\mathcal{R}}$ needs to validate the certificate signature only the first time it receives it from each neighbor. In contrast, $V_{\mathcal{R}}$ generates only one signature per time unit, and for HP specifically it generates one group signature per pseudonym lifetime.

$N$ nodes $V_{i}, i=1, \ldots, N$, produce messages at an aggregate rate $\lambda$, and $V_{\mathcal{R}}$ processes them at a rate $\mu . \lambda$ depends on the number of neighbors, $N$, the message generation rate, $\gamma$, the type of generated messages, and the reliability of communication across the wireless channel. $\mu$ depends on the choice of security primitives (and their security level) and the available on-board processing power. Thus, $\mu$ is constant for a given system configuration.

We view the system operation as a queue: We are interested in the system stability, which depends on $\lambda, \mu$, and the queuing model. To identify an appropriate model, we characterize the arrival process and demonstrate that it can be closely approximated by a Poisson process. Then, we provide an analytical estimate for the average arrival rate, $\lambda$, and validate it through simulations. Finally, we apply queuing theory results, in order to answer the questions outlined above.

\section{A. Characterization of the Arrival Process}

We simulate the system and collect the message inter-arrival times at some $V_{\mathcal{R}}$, for different setups. Then, we fit known distributions to the empirically obtained data and perform a $\chi^{2}$ test to assess the quality of the fitting ( $\mathrm{p}$-value $=0.05$ ). We 


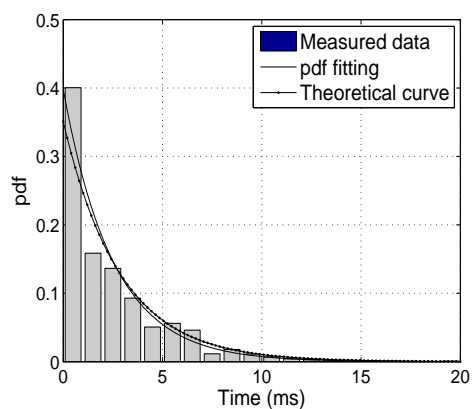

(a) Traffic Arrival Process: Experimental data and Poisson Arrival approximation.

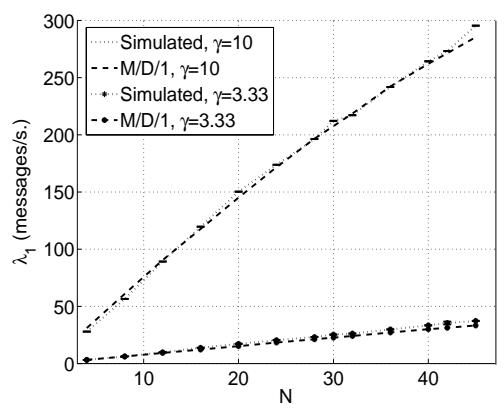

(b) Analytical and simulated $\lambda_{1} ; \alpha=10$ $\beta=0, \tau=60$.

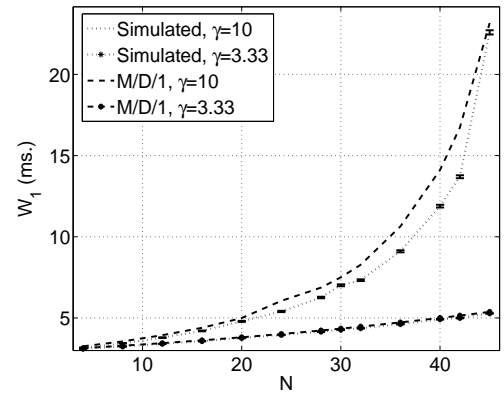

(c) Message verification delay at $V_{\mathcal{R}} ; \alpha=$ $10, \beta=0, \tau=60$

Fig. 3: HP scheme: Comparison between analytical approximation (labeled as $M / D / l$ ) and simulations: Arrival rate, $\lambda_{1}$, and processing delay $W_{1}$, for $S H O R T$ messages, as a function of the neighborhood size $N$.

find that the exponential distribution fits well the empirical data; its memoryless property and the orderliness of packet reception (any node receives one packet at a time and no two or more arrivals occur simultaneously) suffice to approximate arrivals as a Poisson process. Fig. 3(a) shows the collected data and the fitted exponential distribution (superimposed the distribution with the $\lambda$ approximated by Eq. 2 below): the fit is indeed good. This is valid for the type of traffic under consideration, i.e. safety beaconing, which is going to be the majority of the $\mathrm{V} 2 \mathrm{~V}$ traffic. If the type of traffic changes, the arrival process would in principle change.

We assume one processor at $V_{\mathcal{R}}$ with deterministic service times. We consider both $L O N G$ and SHORT packets in the same single queue, with no priority policy and no preemption ${ }^{2}$. The queue is then a multi-class $\mathrm{M} / \mathrm{D} / 1$, in this case with two classes. The average waiting time, $W_{i}$, is given by [32]:

$$
W_{i}=t_{i}+\frac{\sum_{j=1}^{r} \lambda_{j} t_{j}^{2}}{2(1-\rho)}
$$

where $W_{i}, \lambda_{i}$ and $t_{i}$ are the total time in queue, the arrival rate and the service time of the $i$-th (out of $r=2$ ) classes respectively, $\rho=\sum_{i=1}^{r} \rho_{i}$ and $\rho_{i}=\lambda_{i} t_{i}$. The queue length, $L_{i}$, can be derived from Eq. 1 and Little's law [33].

\section{B. Estimation of $\lambda$}

An estimate for $\lambda_{1}$, the arrival rate for SHORT messages (derived in the Appendix) is:

$$
\lambda_{1}=N P\left(1-(1-P)^{K}\right)
$$

with $N$ the number of neighbors, $P$ the average reception probability for messages (beacons), and $K=\frac{\lfloor\tau \gamma / \alpha\rfloor}{2}$. We focus on SHORT packets because they are the majority of the processing load, as explained above and in [9]. From the description of the BP and HP schemes (with Optimizations), the simulations, and the derivation, it appears that $L O N G$ messages have a limited impact.

In Fig. 3, we plot the analytical and simulation results, for $N$ ranging from 4 to 48 vehicles and $\gamma=10$ or $\gamma=3.33$

\footnotetext{
${ }^{2}$ We adopt this as a baseline approach; other policies and system models could be employed.
}

beacons/s; we average over 1000 randomly seeded simulations. Fig. 3(b) shows how many packets $V_{\mathcal{R}}$ must process as a function of $N$, and that this relation is almost linear. As Table IV shows, 333 signature verifications (SHORT packets) per second is the maximum the node we consider here can handle: for $\lambda_{1} \geq 333 \mathrm{msg} / \mathrm{s}$, the node would be unable to keep up and its queue of messages would grow fast. We observe that for $\gamma=10$ (most frequent in the literature), $\lambda_{1}$ increases fast towards this threshold, and the message processing delay, $W_{1}$, increases fast with $N$ (Fig. 3(c)).

Consider an example to illustrate this: with 80 transmitting vehicles in range of $V_{\mathcal{R}}, \alpha=10, \beta=0, \gamma=10$, and $\tau=$ 60 , and the resultant $P=0.655$ (Fig. 2(a)). We assume a highway scenario and a simple content-based optimization: $V_{\mathcal{R}}$ processes a beacon if it comes from a neighbor moving in the same direction (stream of traffic). For simplicity, if the two parts of the road are equally balanced, we consider $N=40$ vehicles out of the 80 neighbors in range. From Eq. 2 we obtain $\lambda_{1}=264.3 \mathrm{msg} / \mathrm{s}$ and from the simulation of the same scenario, the arrival rate would be $259.7 \mathrm{msg} / \mathrm{s}$.

\section{TRANSPORTATION SAFETy AND EFFICIENCY}

We investigate how security affects transportation safety in two settings. First, we consider pairs of vehicles: one is in a dangerous condition transmitting an EBN message and the other is approaching, is previously unaware of the transmitting vehicle and must receive the EBN message. We analyze a fundamental metric, the ability to be notified early: We capture this as the distance at which the receiver is first able to validate the safety messages. The second setting we consider is more involved: We study the occurrence of collisions among vehicles in a platoon of one hundred vehicles, with and without the use of security; the latter serves clearly as a benchmark. We also investigate the impact of penetration rate of vehicular communication rate, to gain insight on how security affects the effectiveness of VC systems, and the EBN application in an urban setting. In terms of transportation efficiency, we investigate the performance of the DFCD application, notably the delay needed to float the information and the distance covered from the location of the event generation. 
Here we integrate the results obtained in the previous sections; we assume that vehicles have sufficient processing power and are able to verify the signatures on all incoming packets. We average over 1000 randomly seeded simulations and present results with $95 \%$ confidence intervals. Recall that Table I summarizes parameter values.

\section{A. Simulation Setup}

We consider four-, six- and eight-lane scenarios, with vehicles placed in two opposing two-, three- and four-lane flows of traffic, respectively. This corresponds to a neighborhood $N$ of 80,120 and 160 vehicles respectively. Vehicles are 4-meters long and are initially uniformly randomly placed along each lane, with an average vehicle-to-vehicle distance of $s$ meters. We focus on one lane of traffic within such a neighborhood of $N$ vehicles, which changes mildly because of mobility. In the two-vehicle setting, there is a small initial "gap" in one lane, depending on the initial spacing of the pair of vehicles; e.g., when they are at 200 meters, there are initially 10 vehicles less present, or in other words 70 (110 or 150) vehicles instead of 80 (120 or 160). The vehicle velocities are initially random with an average $v$, unless stated otherwise; velocities are adapted according the VC system functionality and, in the platoon setting, upon visual contact with the preceding vehicle's brake lights. Vehicles do not change lanes during the simulation, and they process messages originating from vehicles in the same traffic flow (i.e., with the same heading).

We consider an Emergency Braking Notification (EBN) application, with one vehicle in an emergency situation that brakes and starts the transmission of EBN messages. Braking has two effects: (i) it turns on the vehicle's rear red lights that visually warn drivers within range of sight (which depends on the simulated weather conditions), and (ii) it triggers the transmission of EBN warning messages. Besides warning other vehicles, an EBN-warned vehicle warns its driver to start braking shortly afterwards. We model driver reaction times as a result of VC-enabled and visual warnings, with a random variable uniformly distributed between 0.75 and 1.5 $s$. We model weather conditions by setting vehicle braking capabilities and visibility conditions; for example, on a wet road, braking is possible at a rate of $4 \mathrm{~m} / \mathrm{s}^{2}$ and a driver can see up to $30 \mathrm{~m}$. Our simulation conditions agree with related transportation engineering work, e.g., [34], [35].

We also consider a Decentralized Floating Car Data (DFCD) application [36], concerned with how effectively data generated by one vehicle can propagate to an area and a platoon. We consider a highway scenario (platoon of 100 vehicles, with average velocity of $110 \mathrm{Km} / \mathrm{h}$ and average spacing of $30 \mathrm{~m}$ ), with the first (heading) vehicles in the platoon detecting a situation (e.g., traffic jam, road work, etc.) and then starting broadcasting a related notification. Each receiving vehicle will relay a notification with the same content as long as it receives it from at least three distinct vehicles. We consider the DFCD running in parallel with the safety beaconing, and piggy-back DFCD notifications on beacons. With 100 randomly seeded runs of $20 \mathrm{sec}$ of simulated time (after the warm-up of 60 sec), we study the effect of security on three parameters: (i) the time for the notification to reach $90 \%$ of the platoon, (ii) the time for it to "cover" a distance of $1000 \mathrm{~m}$ from the origin of the vehicle first detecting the situation (event), and (iii) the maximum distance the notification propagated.

Two-Vehicle Setup: We consider one transmitter, $V_{t}$, and one receiver, $V_{\mathcal{R}}$, at an initial distance of $d$ meters, with $V_{\mathcal{R}}$ always behind $V_{t}$ in the same lane and with a velocity for $V_{\mathcal{R}}$ higher than that of $V_{t}$. $V_{\mathcal{R}}$ moves at a constant relative speed $\Delta v$ with respect to $V_{t}$, without any other vehicle in between. For simplicity, we elect $s$ to be such that it is less than or equal to the nominal communication range at the beginning of each simulation. We choose two setups, one with $\Delta v=20$ $\mathrm{Km} / \mathrm{h}$ and $s=150 \mathrm{~m}$, and the other with $\Delta v=35 \mathrm{Km} / \mathrm{h}$ and $s=200 \mathrm{~m}$. We evaluate how the optimization parameters, $\alpha$ and $\beta$, affect the distance, $D$, at which $V_{\mathcal{R}}$ receives the first $K_{V_{t}}^{i}, \Sigma_{C A, V}\left(K_{V}^{i}\right)$ from $V_{t}$. In this setting, we wish to test the ability of the secure VC system to deliver safety information, especially under challenging conditions; e.g., $V_{\mathcal{R}}$ is very close to $V_{t}$ when the latter changes to a new pseudonym (and private key). Therefore, in order to evaluate the VC performance alone, we do not consider the rear red lights of $V_{t}$; which, would naturally warn the driver of a $V_{\mathcal{R}}$ in line of sight.

Vehicle Platoon Setup: We focus on a platoon of one hundred cars along a single lane moving with similar velocities, denoted as $V_{1}$ to $V_{100}$, with $V_{1}$ for the vehicle at the front and $V_{100}$ at the rear of the platoon. We utilize values of $s=20$ $m$, velocities on the average $v=80 \mathrm{Km} / \mathrm{h}$. We analyze how many collisions occur when the leading vehicle $V_{1}$ brakes for an emergency and starts sending EBN messages. Once some $V_{i}$, with $i>1$ receives the warning, it starts sending EBN messages itself. As proposed in [37]-[39], when $V_{i}$ receives a warning from a $V_{j}$ with $j>i$, it stops transmitting warnings, because it assumes that at least one vehicle behind $V_{i}$ has already been warned. In this setting, we consider rear red-light warnings. For the urban environment, we consider multi-lane settings and platoons of vehicles that move more slowly and are closer to each other than in the highway settings (average velocity of $45 \mathrm{Km} / \mathrm{h}$ and a vehicle distances of $10 \mathrm{~m}$, on a wet road). We choose pseudonym lifetime $\tau=60 \mathrm{~s}$. We consider the first $60 s$ of the simulation time as a warmup period, during which no emergency conditions arise. This approximates a realistic situation: When an emergency arises, vehicles have already validated (identified) some of their neighbors and can thus immediately accept their warnings. The simulation concludes when all vehicles in the platoon are immobile, with $V_{1}$ not moving further after its emergency braking.

First, we consider scenarios where all vehicles are equipped with VC systems. Intuitively, full VC deployment can lead to better safety, but it also corresponds to more strenuous conditions (processing and communication overhead). Nonetheless, VC will be deployed gradually, over a period of several years. Thus, we define the penetration rate, $\mathrm{pr}$, as the fraction of VC-enabled vehicles, and we analyze the system behavior as a function of $p r$. Equipped vehicles behave as described above, whereas non-equipped vehicles rely only on visual means (the red lights of the preceding vehicle) to detect emergencies. We analyze this scenario with 4 lanes of traffic, $\alpha$ equal to 1 or 


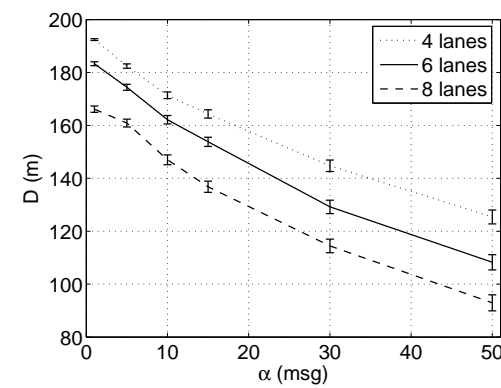

(a) Reception distance, $D$, as a function of $\alpha$; $s=200 \mathrm{~m}, \Delta v=35 \mathrm{Km} / \mathrm{h}$.

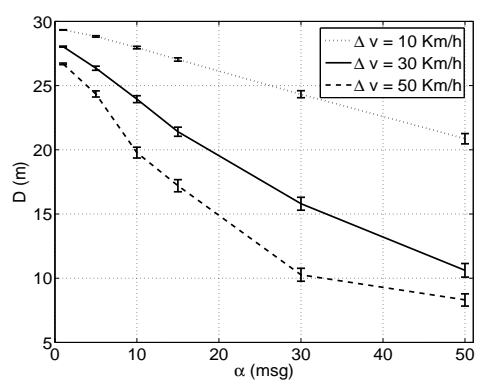

(b) Reception distance,
$\alpha ; s=30 \mathrm{~m}, 8$ lanes.

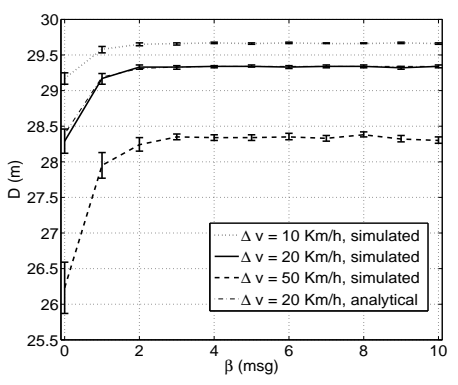

(c) Reception distance, $D$, as a function of $\beta$; $\Delta v=20 \mathrm{Km} / \mathrm{h}, \alpha=10,8$ lanes.

Fig. 4: Distance $D$, at the time of reception of the first certificate at the trailing vehicle, $V_{\mathcal{R}}$, as a function of $\alpha, \beta$.

$10, \beta$ to $0, \gamma$ to 10 or 3.33 beacons/s, and $p r$ from 0.05 to 1 .

\section{B. Two-Vehicle Simulation}

Impact of pseudonym change on safety: In Fig. 5(a), the distance $D$ at which $V_{\mathcal{R}}$ receives the first certificate $\Sigma_{C A, V}\left(K_{V}^{i}\right)$ decreases as the Certificate Period $\alpha$ increases: If a $L O N G$ message from $V_{t}$ is missed, $V_{\mathcal{R}}$ has a chance to receive the next one only after $\alpha$ additional beacons from $V_{t}$. Nonetheless, we observe that messages from $V_{t}$ can be validated in all cases before the distance becomes dangerously small.

Missing a new pseudonym could be dangerous if $V_{\mathcal{R}}$ (and in general any vehicle) is close to $V_{t}$ at the time of pseudonym change and has high positive relative speed (i.e., approaches fast $V_{t}$ ). To capture such situations, we fix $s=30 \mathrm{~m}$ and we vary $\Delta v=10,20$ and $50 \mathrm{Km} / \mathrm{h}$ (Fig. 4(b)): The effect of $\alpha$ remains, but we also observe that with a higher $\Delta v$, the drop in the reception distance with $\alpha$ is faster. Overall, pseudonym switching can be risky if it happens when vehicles are close to (fast approach) each other.

Optimization 3, not used so far, can address this problem, as shown in Fig. 4(c), for $\alpha=10$ and with $\beta$ varying from 0 to 10 . Even a single "pushed" message $(\beta=1)$ enables reception within roughly 2 meters after the pseudonym change, regardless of speed and initial distance (clearly, the actual reception distance depends on those parameters). Increasing redundancy, that is setting $\beta$ beyond 3, does not improve robustness any further. We observe in Fig. 4(c) that even $\beta=1$ is near-optimal.

Intuitively, this is because the probability of receiving a $L O N G$ message when $V_{\mathcal{R}}$ and $V_{t}$ are very close to each other is relatively very high. If these two nodes are far apart, the probability of successful reception is relatively low, but there will be several opportunities (subsequent transmissions) for $V_{\mathcal{R}}$ to receive a $L O N G$ message from $V_{t}$. More precisely, let $Z$ be a discrete random variable for the slot in which a $L O N G$ message is first received (during the lifetime of the pseudonym). If $E(Z)$ is the mean value, the average distance from $V_{t}$ that the first $L O N G$ is received by $V_{\mathcal{R}}$ is $D=E(Z) \gamma^{-1} \Delta v$. $V_{t}$ transmits $L O N G$ packets for $\beta$ consecutive slots, and every $k \alpha$ slots. The probability that a $L O N G$ packet is first received at some $j$ slot is simply the probability it is not received at any of the $i<j$ slots and it is received at the $j$-th, where $i, j$ take values from $I=1,2, \ldots, \beta, \beta+1, \ldots, \beta+1+\alpha, \ldots$ :

$$
P_{j}=P(j) \prod_{k \in I}(1-P(k))
$$

The probabilities at each slot, $P(k)$, differ, as they depend on the distance of the two nodes (and their neighborhood more generally). Using values for different distances obtained experimentally (Sec. VI and [9]), we compare Eq. 3 to the simulation results, for $s=30 \mathrm{~m}, \Delta v=20 \mathrm{Km} / \mathrm{h}, \alpha=10$, 8 lanes of traffic and $\gamma^{-1}=100 \mathrm{~ms}$. Fig. 4(c) shows $D$ calculated with the help of Eq. 3 and the experimental data.

\section{Platoon Analysis}

1) Fully deployed VC: Fig. 5(a) shows the percentage of vehicles, within a platoon of one hundred, which crash as a function of $\alpha$. As a reference, we simulate the VC system and EBN application without security. In the absence of V2V communications, $80 \%-100 \%$ of vehicles crash; for the same scenarios, safety messaging reduces the number of crashes to approximately $10 \%$ of all vehicles. Then overall, as expected, security increases crashes compared to unsecured VCs: the increased network overhead and protocol restrictions on which alert messages can be validated delay the reception of valid EBN messages. ${ }^{3}$

However, we observe that the secure VC protocol parameters affect the safety application. We observe first a decrease in the average fraction of crashes, as $\alpha$ increases, and then a slow increase as $\alpha$ increases further. This is due to two competing factors: The increase of $\alpha$ reduces the channel load and thus increases the per-packet reception probability, but the authentication delay for a receiver missing a $L O N G$ packet also increases; e.g., for $\alpha=50$, the authentication delay is (at least) $5 \mathrm{~s}$. Optimization 3 reduces the number of crashes with respect to the non-optimized protocol, with the same value for $\alpha$, as it adds negligible overhead but manages to reduce the authentication delay, as explained above.

Fig. 5(b) shows the percentage of vehicles that collide in an urban setting. Again, we observe that security has a limited

\footnotetext{
${ }^{3}$ For non-secured $\mathrm{VC}$, the $\mathrm{x}$-axis, the $\alpha$, is not a parameter that affects its operation. This is why the corresponding curve is essentially flat, with minor variability due to the randomly seeded simulation scenarios.
} 


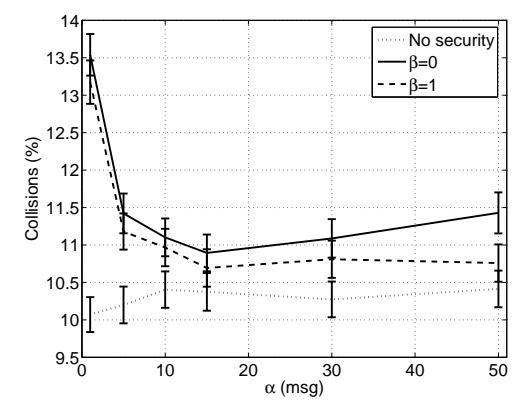

(a) Collisions as a function of $\alpha, \beta ; 8$-lane highway; comparison with unsecured VC.

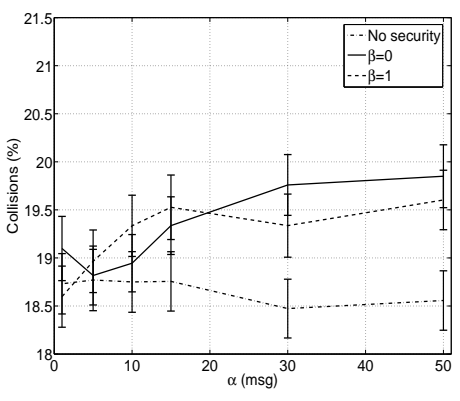

(b) Collisions as a function of $\alpha, \beta$; u setting; comparison with unsecured VC.



(c) Collisions as a function of pr; 4-lane highway

Fig. 5: Transportation safety with secure VC: Collisions as a percentage of vehicles, in an emergency situation; varying road conditions, message rates, protocol parameters, and VC technology penetration rate; HP scheme, $\gamma=10$ beacons/s.

effect on the non-secured EBN application performance. The difference from the highway scenario is the way security parameters affect performance. This is a dense VC network, but vehicles move with lower velocities and they are more densely packed. The lower inter-vehicle distances lead to higher reception probability, even though the channel remains relatively heavily loaded; thus, $\beta$ does not give a significant advantage. Low inter-vehicle distances (high reception probability) result in an increase in vehicle collisions (crashes) only for high $\alpha$ (higher authentication delay). This is analogous to the highway observations; the difference is that low vehicledistances (lower driver reaction margins) counter balance low velocities.

2) Effect of secure VC penetration rate on safety: Fig. 5(c) shows what happens in a scenario, with average vehicle spacing of $40 \mathrm{~m}$, speed of $65 \mathrm{Km} / \mathrm{h}$, braking capability of 6 $\mathrm{m} / \mathrm{s}^{2}$ and visual range of $70 \mathrm{~m}$ (modeling dry road conditions and good weather). First, we observe a percentage of crashes between $25-30 \%$ and none (lower than that for the earlier more challenging scenarios). The curves for different $\gamma$ values are well separated, with more crashes for $\gamma=3.33$, which indicates that this beacon frequency would not be sufficient to warn drivers (although the reception reliability is higher (Fig. 2(a)). Tuning $\alpha$ affects the number of crashes when $p r$ is in the range of $40-80 \%$; for $\alpha=10$ we observe an increase variable from 15 to $40 \%$ compared to the case with $\alpha=1$. Conversely, if VC has relatively low $(p r<0.4)$ or high $(p r>$ 0.8 ) penetration, security optimizations have a limited impact.

3) Effect of secure VC on efficiency applications: Fig. 6 shows the communication performance for the DFCD application, operating on top of, thus being constrained by, the more resource-intensive safety beaconing. ${ }^{4}$ We observe that security parameters affect the performance. More so for $\alpha$, which yields the best performance for all three metrics for values close to $\alpha=10$. Increasing $\alpha$ reduces communication overhead, increases reliability, and reduces processing delays. For high $\alpha$ values (e.g., above 20), there is a mild deterioration performance due to the increase in authentication delay.

\footnotetext{
${ }^{4} \mathrm{We}$ do not measure the effect the transportation efficiency application has (e.g., on average trip times) as this would require a global system consideration.
}

\begin{tabular}{|l|c|c|c|}
\hline & BP & GS-I & GS-II \\
\hline RL size & $144 R \mathrm{~KB}$ & $32 R \mathrm{~B}$ & $32 R \mathrm{~B}$ \\
Revocation check cost & $1 \mu \mathrm{s}$. & $15 R \mathrm{~ms}$. & $30 \mathrm{~ms}$. \\
Hash table construction & $0.1 R \mathrm{~s}$. & - & $1.5 R \mathrm{~s}$. \\
Memory requirements & $140 R \mathrm{~KB}$ & - & $38.4 R \mathrm{~KB}$ \\
\hline
\end{tabular}

TABLE VI: Indicative values for revocation check costs as a function of $R$, for $C_{p}=15 \mathrm{~ms}, k=100, \tau=60 \mathrm{~s}$.

Intuitively, the parameter values affect how the network can relay the DFCD notifications. If nodes do not have the credentials of the neighbors when they receive a notification, especially as they need at least three distinct valid notifications, they will be slow in relaying it. The effect of $\beta$ relates to that: with less frequent retransmissions of LONG packets, loss upon a pseudonym change would incur further delay. $\beta=1$ remedies this, keeping stable performance for all three metrics, even for high $\alpha$.

Comparing the secured to the unsecured VC system DFCD performance, we see tangible deterioration because of security: The same maximum area is covered (with an approx. $1.5 \%$ deterioration only), but with approximately $42 \%$ higher delay to cover $1000 \mathrm{~m}$ behind the event (in the sense of the traffic flow), and approximately $30 \%$ higher delay to cover $90 \%$ of the platoon nodes. This is because the scenario is challenging (multi-lane, heavily loaded setting), exacerbated by the need to have 3 distinct copies of the DFCD notification. With the expected latency per hop varying per DFCD message type, but typically in the order of $500 \mathrm{~ms}$, we see that delays of $1.2 \mathrm{sec}$ for $1000 \mathrm{~m}$ (approx. five hops in our setting) for the secure VC system are satisfactory; so is the delay for the $90 \%$ platoon coverage (approx. $3 \mathrm{~km}$ in our setting), and the maximum covered area (the total of the platoon vehicles). ${ }^{5}$

\section{REVOCATION}

Here we discuss the revocation costs, based on the use of Revocation Lists (RLs). This is a largely orthogonal problem to

\footnotetext{
${ }^{5} \mathrm{We}$ did not consider here propagation of the information by vehicles in the opposite traffic flow, or geocasting, techniques assumed in place in order to achieve expected areas of coverage in the order of 10 or $20 \mathrm{~km}$ (depending on the nominal communication range).
} 


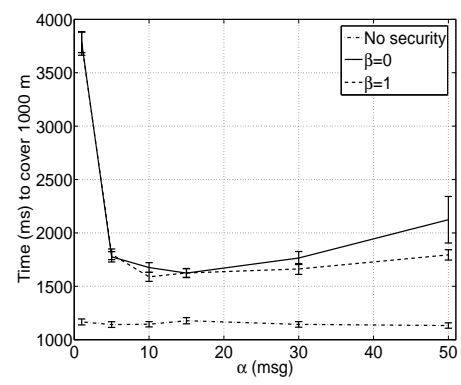

(a) Delay (ms) to cover 1000 meters from a new event (notification).

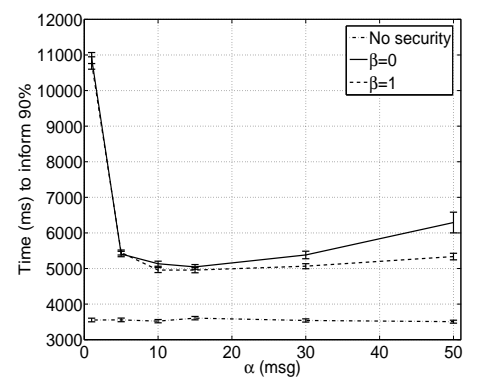

(b) Delay (ms) to cover $90 \%$ of a 100 -vehicle platoon, after a new notification (event).

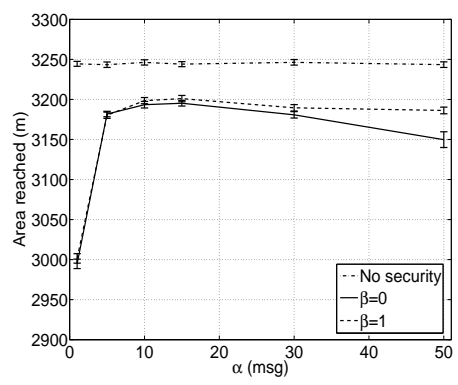

(c) Area reached by a new notification (event).

Fig. 6: Decentralized Floating Car Data (DFCD); communication performance.

this investigation and out of the scope of this paper; moreover, there are several unknown parameters and factors in terms of the instantiation of a revocation solution. Nonetheless, in order to provide a complete picture, we consider the revocation overhead for each of the security schemes considered. We did not consider revocation in Sec. VII, but we do this in Sec. X: it suffices to add the revocation processing delay to the one for validating $L O N G$ messages (the first-received $L O N G$ per node and $\tau$ for BP and HP).

The basic difference between BP and HP (and GS) schemes is that the former deals with short-term keys and the latter with long-term ones. The number of vehicles that would be revoked is not currently known and it is hard to estimate, because it would depend on policy decisions, the size of the system in each region, among other currently unknown aspects. Here we denote the number of revoked vehicles as $R$. Then, for the HP (and GS) scheme, the size of the revocation list would be $\left|R L_{H P}\right|=R$. Whereas, for $B P,\left|R L_{B P}\right|=c \times R$, where $c$ is essentially the number of temporary keys each vehicle holds at the time of its revocation.

$c$ can be a large number, in the order of $10^{4}$ to $10^{6}$; but, again, its actual value depends on factors out of the scope of this paper: for example, the ability of vehicles to have frequent access to a trusted third party to obtain their shortterm certificates, or the autonomy of vehicle policies mandate. We can consider that $c$ corresponds approximately to the number of pseudonyms a vehicle obtains at a "pseudonym refill", and we can assume that pseudonyms are valid only between two consecutive refills. Then, a revoked node running BP would be unable to obtain a new set of pseudonyms [14], and $R L_{B P}$ would include only the pseudonyms granted at the last refill. Consider an example for $c$ : with $\tau=60 \mathrm{~s}$, in one day a vehicle $V$ would "consume" 1440 pseudonyms. Assuming that pseudonym refills take place once per month, then $c=43200$; if the refills were made once per year, then $c=518400$. In the rest of this paper, to provide illustrative examples, we assume that on the average $c \approx 10^{4}$.

The cost to verify whether a pseudonym is revoked is the cost of a lookup into the $R L$. This can be achieved in constant time, e.g. by using a hash table. In this case, the construction of the data structure is proportional to $\left|R L_{B P}\right|$, and it must be performed every time a new $R L_{B P}$ is received. The required memory is also $\approx\left|R L_{B P}\right| \times E_{B P}$ bytes, where $E_{B P}$ is the size of one entry in the hash table. $E_{B P}$, composed by a serial number and a revocation date, is 14 bytes [1]. Thus, for each revoked vehicle (i.e. long-term identity) with $c$ pseudonyms, at least $140 \mathrm{~KB}$ would be needed.

For GS and HP schemes, we extract relevant data from [22], [27]. Each entry in $R L_{H P}$ is a revocation token of 32 bytes (Note: for simplicity, we use interchangeably the subscript GS for HP in terms of revocation.). Then, we consider two related revocation methods proposed in [22]: the first one, we term $G S-I$, incurs processing costs proportional to the RL size; the second one, GS-II, has fixed costs independent of the RL size, but it might allow the linking of some Group Signatures [22].

GS-I: The revocation tokens, each $E_{G S}=32$ bytes, are used directly for the revocation check process. The cost to verify one entry is $C_{p} \times\left|R L_{G S}\right|$, where $C_{p}$ is the cost of computing one bilinear map. Group signatures of not-revoked nodes cannot be linked under any circumstances, but checking if a signer is revoked requires a traversal of the entire $R L_{H P}$ (in other words, it is linear in the number of revoked vehicles).

GS-II: The basic difference from $G S-I$ is the calculation of the Group Signatures, which include some intended recipient, $S$, a random positive integer $r$, now chosen by the signer to have a value less than a security parameter $k$. As it will be explained in further detail below, this construction allows $S$ to pre-compute $k$ revocation values and check the revocation status of the signer through a simple look-up; if, however, $k$ were low, the signer might be forced to re-use $r$ values, and these group signatures from that signer could be linked.

As the safety beacons are broadcasted, we need to adapt the scheme to the VC context: We redefine $S$ to be $S=\langle G, T\rangle$, where $G$ indicates a geographical area and $T$ a time interval. Essentially, in a given area and time, every recipient can perform the fast revocation status check. For practical reasons, $G$ and $T$ can be coarsely defined, so that receiving nodes can easily determine the appropriate values (e.g., with the help of their on-board clock, GPS receiver, or other localization means with the help of terrestrial infrastructure).

Upon reception of a new $R L_{G S}$, a verifier $V_{\mathcal{R}}$ in $S$ precomputes and stores the $k$ revocation values for each entry in the $R L_{G S}$, at a cost of $2 C_{p}$ per entry. The cost to build this data structure is then $2 C_{p} \times R \times k$. Upon receipt of a $\Sigma_{C A, V}\left(K_{V}^{i}\right)$, the verifier $V_{\mathcal{R}}$ performs a lookup into the table and if no match is not found (i.e. the signer is not revoked) 


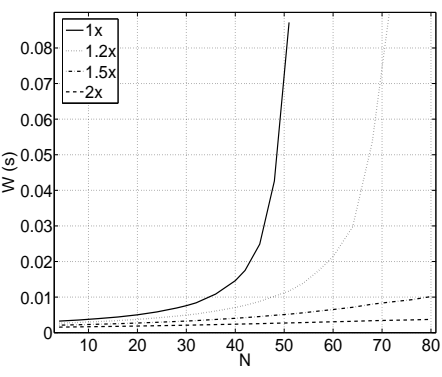

(a) HP.

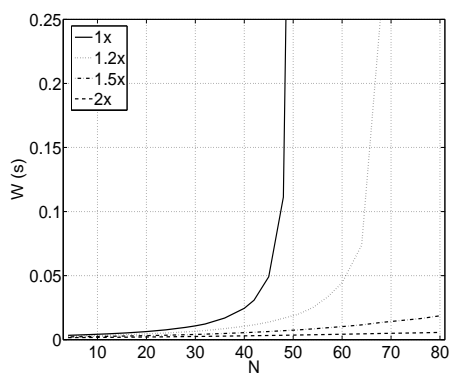

(b) HP with revocation check (GS-II).

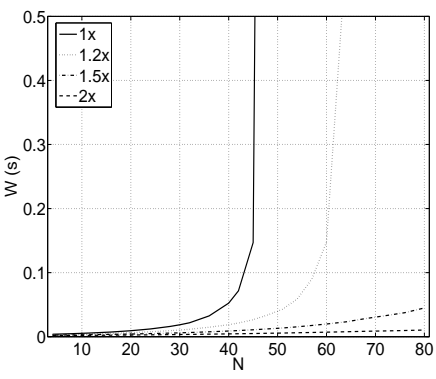

(c) HP enhanced with time-limited GS.

Fig. 7: Processing delay in future secure VC systems, with processing acceleration, as a function of network size.

it validates the signature. The cost to verify if the sender is revoked is $2 C_{p}$, plus the lookup cost, which is negligible compared to $C_{p}$. The memory needed is approximately $\left|R L_{G S}\right| \times k \times E_{G S}$ bytes. $E_{G S}$ is the result of one pairing computed from the revocation token, and its size is 384 bytes.

For a given $S=<G, T>$, the value of $k$ should be chosen such that a single sender is not forced to use the same $r$ twice or more. Basically, it should be $k \geq\lceil|T| / \tau\rceil$. However, high $k$ would increase the pre-computation costs, which also depends on how the $R L_{G S}$ changes over time. Investigating trade-offs due to chosen values, e.g., $\tau,|T|$, is left as future work. In order to provide a numeric example, we fix $k=100$, which corresponds to $|T|=1 \mathrm{~h} 40 \mathrm{~min}$, and we summarize the results in Table VI, assuming $C_{p}=15 \mathrm{~ms}, k=100, \tau=60 \mathrm{~s}$, and the basic operations on the hash table, such as memory copy and data lookup, to be $1 \mu \mathrm{s}$.

Clearly, the BP scheme incurs the minimum computational overhead but it has by far the longest RL. Moreover, the GSI method could be cumbersome to apply, especially for the platform we considered here and for sizeable RLs. Then, the cost of GS-II is independent of $R$ but it remains higher than that of BP. Nonetheless, GS-I and GS-II have the advantage of much smaller RLs, and thus require lower memory and bandwidth for storage and transmission.

Finally, recall that for the GS scheme, the revocation status check must be performed for each message; whereas for the HP scheme, the check is needed only once per previously unseen pseudonym. It is also important to note that the revocation of a node implies its anonymity is lost; then, any entity that has a transcript of its past transmissions in a given area, can use the corresponding revocation token and identify which messages in the transcript were sent by the revoked node.

\section{Discussion And Future Work}

The introduction of anonymous authentication, for infrequent operations, as in HP, offers the advantage of simpler administration (much lower number of credentials, on-the-fly generation), but it increases processing cost. This is so for each GS-related operation (LONG packets for HP). Anonymous authentication brings forth another challenge: a misbehaving node could abuse its anonymity, generate and sign multiple pseudonyms, and thus appear as multiple nodes, also known as a Sybil node behavior.
Each legitimate node that monitors its neighborhood and relies on its own sensor readings (e.g., infra-red or radar transceivers) could weed out such Sybil identities (pseudonyms), as pointed out in [40], or it could detect other inconsistencies, e.g., between the apparent density vehicles (inflated by a Sybil node) and the average velocity (in principle dropping with increased vehicle density). But there are proactive defenses to contain Sybil misbehavior. First, the invocation of the GS primitive can be regulated by trusted hardware, e.g., the Hardware Security Module (HSM) of the $\mathrm{SeVeCom}$ architecture [2]. A different approach is that of utilizing primitives that limit the number of GS generations per unit of time;.e.g, [41] can be set to allow each node to generate one GS per pseudonym lifetime. If a Sybil node exceeds this, any such GS (and thus pseudonym) will be linkable by any receiving correct node.

The addition of new features, e.g, a time-limited GS generation, will have additional resource requirements. This is a more general concern. But it is natural to expect that onboard processing capabilities will also improve. For example, there are already secure $\mathrm{VC}$ boxes that achieve nearly a ten-fold increase (www.escrypt.com) in terms of processing (thanks to cryptographic hardware acceleration) compared to our benchmark platform.

In Fig. 7, we consider processing delay for various processing acceleration factors over our benchmark platform. A factor $x$ decreases processing delay per cryptographic operation by $1 / x$. We demonstrate how our model of Sec. VII can yield resource provisioning as the scale increases. Fig. 7 shows that in order to keep the per-safety-beacon delay flat for a large neighborhood (160 vehicles within range, verification from 80 ones), $x=2$ is necessary. This is sufficient even for real-time revocation (i.e., 30ms delay for each LONG for the benchmark platform, and $15 \mathrm{~ms}$ for $x=2$ ), or time-limited GS scheme (e.g., for [41], we approximate that a time-limited GS-verification delay is approximately 2.5 times than of the "plain" GS). Note the progressive increase of the delay (y-axis) and recall that $x$ speeds up not only the relatively infrequent LONG processing but also the majority of the lower-cost SHORT processing.

Future work will include additional characteristics of the transportation environment, e.g., traffic lights, change of routes other safety applications (corner-collision avoidance), alter- 
native communication technologies, and any roles of infrastructure. Alternative security and privacy enhancing mechanisms is another future work direction, including alternative cryptographic primitives and functionality and further implementations and benchmarking. A different but related issue: general privacy considerations, across the data-handling "ecosystem", beyond the mostly location-privacy related VCcentric considerations.

\section{RELATED WORK}

The use of pseudonyms was first envisioned in [42] and more recent works considered their use in the context of VCs, e.g., [14], [43], [44]. More generally, several recent works are concerned with different aspects of security and privacy of vehicular networks: They either outline challenges [45], [46], describe particular attacks [47], [48] or more general attack overviews [49], [50], propose mechanisms [40], [44], [51], [1], and schemes for revocation [2], [13], [52]. [53], and the recent follow-up [54] that provides performance evaluation results, combine public and symmetric key cryptography to authenticate messages and is complementary to our work.

The idea of pseudonym self-generation for ubiquitous computing is proposed, independently of our work, in [55]. More recently, [56] applied that crypto-system to VANET. These works do not consider all the system-level issues we consider in this work, such as certificate distribution and application robustness. Our findings and mechanisms also apply to their work, complementing and extending it.

An alternate approach to reducing packet overhead and computation efforts is presented in [57]: it proposes that a signer attaches its certificate to messages only when it detects a change in its neighborhood, with such changes detected from beacons. In dense topologies, the results of [57], although obtained in less realistic conditions, are comparable to ours.

Two recent works propose to use bilinear pairings to provide privacy in VANET. The approach of [58] is similar to our GS scheme, thus it would be cumbersome if not impossible to apply for safety beaconing. [59] employs a mix of traditional public-key cryptography and bilinear pairings; this bears some resemblance to our HP scheme, but it is mainly limited by its strong reliance on the presence of RSUs, not envisioned to be densely present in most, if not all, deployments.

A few other papers [30], [37]-[39] propose and analyze VCbased algorithms for transportation safety, and transportation engineers studied how to reduce car collision chains [34], [35]. But the combined study of transportation safety applications enabled by VC and the effect of security overhead was not considered.

\section{CONCLUSION}

We have analyzed the effect of security on the VC system effectiveness, notably a safety and an efficiency application. We have provided a framework to analyze the performance of secure VC systems, along with schemes that reduce the complexity and the overhead of security, we considered multiple system operation dimensions and identified interdependencies of various factors. We strongly believe that systematic evaluation of the overall performance is critical, especially for pervasive computing systems that are tightly coupled to their users. As security and privacy are paramount for those systems, yet they incur significant overhead, designs should be validated, to show that the secured systems can be effective as envisioned and needed. We do this for a system as complex as VCs: This being the first work taking this approach, we aspire to produce and see further results for a technology that can be very widely deployed in the near future.

\section{APPENDIX}

We derive here the approximation for $\lambda_{1}$, the average arrival rate for $S H O R T$ messages, i.e. Eq. 2 in Sec. VII. We consider a set of $N$ transmitters $V_{i}$ and one receiver $V_{\mathcal{R}}$, all running the protocol with the same configuration (i.e. all $V_{i}$ use the same beacon interval $\gamma$, the same pseudonym lifetime $\tau$, and the same Certificate Period $\alpha$, and $\beta=0$ as a minor simplification due to the low effect of $\beta$ on overhead for the values recommended by the findings in Sec. VIII). As discussed in Sec. VIII and Sec. VI, each message is received with a different probability depending not only on the overall setup but also on the (fast changing) distance between sender and receiver; obtaining these values is far from trivial.

Here, we make a few simplifying assumptions: We consider some slot, $t$, and assume that all $N$ transmitters send a beacon, $S H O R T$ or $L O N G$ during that slot. Recall that each $L O N G$ beacon entails a calculation equivalent to a $S H O R T$ beacon (due to the verification of the ECDSA signature), plus an additional overhead when $V_{\mathcal{R}}$ receives a $L O N G$ one with a new pseudonym (due to the GS verification). We assume that each beacon is received with probability $P$, independently from all other $N-1$ beacons. (The probability of reception is for example the average of the probabilities of reception at different distances for the given neighborhood, obtained from Sec. VI).

For some sending node $V_{i}$, its message will be verified by $V_{\mathcal{R}}$ with probability $p \times \operatorname{Prob}\left\{V_{i}\right.$ 's $L O N G$ was already received $\}$. This is determined by how many times the given $V_{i}$ transmitted a $L O N G$ message (as we assume that the reception of $L O N G$ packets is also with probability $P$ ). By the definition of the scheme (HP or BP), during $\tau$ seconds, a pseudonym lifetime, $V_{i}$ transmits $\lfloor\tau \gamma / \alpha\rfloor L O N G$ packets.

The number of $L O N G$ packets from $V_{i}$ that $V_{\mathcal{R}}$ observed (i.e. could potentially receive being in range) depends on various factors beyond this model (e.g., mobility, individual vehicle trajectories, road shape, communication obstructions). Here we make one final simplifying assumption: Each $V_{i}$ is on the average "half-way through" its current $\tau$, thus, it has on the average transmitted $K=\frac{\lfloor\tau \gamma / \alpha\rfloor}{2} L O N G$ beacons.

Then, the $\operatorname{Prob}\left\{V_{i}\right.$ 's $L O N G$ was already received $\}$ is essentially the $\operatorname{Prob}\left\{V_{i}\right.$ 's $L O N G$ was received at least in one of $K$ tries $\}$; this is equal to $1-\operatorname{Prob}\left\{V_{i}\right.$ 's $L O N G$ was received in none of $K$ tries $\}=1-(1-P)^{K}$. As all of the $N$ nodes sent out a packet, in each slot, on average $V_{\mathcal{R}}$ will receive

$$
\lambda_{1}=N P\left(1-(1-P)^{K}\right)
$$

to process. This completes the derivation of Eq. 2 . 


\section{REFERENCES}

[1] IEEE1609.2, "IEEE Trial-Use Standard for Wireless Access in Vehicular Environments - Security Services for Applications and Management Messages," Jul. 2006.

[2] P. Papadimitratos, L. Buttyan, T. Holczer, E. Schoch, J. Freudiger, M. Raya, Z. Ma, F. Kargl, A. Kung, and J.-P. Hubaux, "Secure Vehicular Communication Systems: Design and Architecture," IEEE Comm. Mag., vol. 46, no. 11, Nov. 2008

[3] F. Kargl, P. Papadimitratos, L. Buttyan, M. Müter, B. Wiedersheim, E. Schoch, T.-V. Thong, G. Calandriello, A. Held, A. Kung, and J.-P. Hubaux, "Secure Vehicular Communication Systems: Implementation, Performance, and Research Challenges," IEEE Comm. Mag., vol. 46, no. 11, Nov. 2008.

[4] "NoW: Network on Wheels," uRL: http://www.network-on-wheels.de/.

[5] "The Car-to-Car Communication Consortium." [Online]. Available: http://www.car-to-car.org

[6] "The eSafety eSecurity Working Group." [Online]. Available: http://www.esafetysupport.org/en/esafety_activities/ esafetyworking_groups/esecurity.htm

[7] "DSRC: Dedicated Short Range Communications," http://grouper.ieee.org/groups/scc32/dsrc/index.html.

[8] G. Calandriello, P. Papadimitratos, J.-P. Hubaux, and A. Lioy, "Efficient and Robust Pseudonymous Authentication in VANET,' in VANET '07, Montreal, Canada, Sept. 2007.

[9] P. Papadimitratos, G. Calandriello, A. Lioy, and J.-P. Hubaux, "Impact of Vehicular Communication Security on Transportation Safety," in MOVE '08, Phoenix, AZ, USA, Apr. 2008.

[10] "IEEE P802.11p/D3.0, Draft Amendment for Wireless Access in Vehicular Environments (WAVE)," July 2007.

[11] "Dedicated Short Range Communication at 5.9 $\mathrm{GHz}$ Standards Group." [Online]. Available: http://www.iteris.com/itsarch/html/standard/dsrc5ghz-b.htm

[12] "ISO TC204 Working Group 16." [Online]. Available: http://www.calm.hu/

[13] P. Papadimitratos, G. Mezzour, and J.-P. Hubaux, "Certificate Revocation List Distribution in Vehicular Communication Systems," in VANET '08, San Francisco, CA, USA, Sept. 2008.

[14] P. Papadimitratos, L. Buttyan, J.-P. Hubaux, F. Kargl, A. Kung, and M. Raya, "Architecture for Secure and Private Vehicular Communications," in ITST'07, Sophia Antipolis, France, Jun. 2007.

[15] D. Chaum and E. van Heyst, "Group Signatures." in EUROCRYPT '91, Brighton, UK, Apr. 1991.

[16] G. Ateniese and G. Tsudik, "Group Signatures à la carte," in SODA '99, Baltimore, MD, USA, Jan. 1999.

[17] P. Syverson and S. Stubblebine, "Group Principals and the Formalization of Anonymity." in FM '99, Toulouse, France, Sept. 1999.

[18] E. Brickell, J. Camenisch, and L. Chen, "Direct Anonymous Attestation," in CCS '04, Washington DC, USA, October 2004.

[19] D. Boneh, X. Boyen, and H. Shacham, "Short Group Signatures," in Crypto '04, Santa Barbara, CA, USA, Aug. 2004.

[20] M. Bellare, D. Micciancio, and B. Warinschi, "Foundations of Group Signatures: Formal Definition, Simplified Requirements and a Construction based on Trapdoor Permutations," in Adv. in Cryptology, May 2003.

[21] M. Bellare, H. Shi, and C. Zhang, "Foundations of Group Signatures: The Case of Dynamic Groups," in CT-RSA '05, San Francisco, CA, USA, Feb. 2005.

[22] D. Boneh and H. Shacham, "Group Signatures with Verifier-Local Revocation," in CCS '04, Washington DC, USA, Oct. 2004.

[23] IEEE 1363a-2004, "IEEE Standard Specifications for Public-Key Cryptography - Amendment 1: Additional Techniques," 2004.

[24] "The CVIS project, http://www.cvisproject.org/."

[25] "OpenSSL," http://www.openssl.org.

[26] M. Brown, D. Hankerson, J. Lopez, and A. Menezes, "Software Implementation of the NIST Elliptic Curves Over Prime Fields," in CT-RSA 2001, San Francisco, CA, USA, April 2001.

[27] N. Koblitz and A. Menezes, "Pairing-Based Cryptography at High Security Levels," Cryptology ePrint Archive, Report 2005/076, 2005.

[28] M. Nakagami, "The m-distribution, a General Formula of Intensity Distribution of the Rapid Fading," in Statistical Methods in Radio Wave Propagation, W. G. Hoffman, Ed. Oxford: Pergamon, 1960.

[29] Q. Chen, F. Schmidt-Eisenlohr, D. Jiang, M.-T. Moreno, L. Delgrossi, and H. Hartenstein, "Overhaul of IEEE 802.11 Modeling and Simulation in ns-2," in MSWiM '07, Chania, Greece, Oct. 2007.

[30] Q. Xu, T. Mak, J. Ko, and R. Sengupta, "Vehicle-to-Vehicle Safety Messaging in DSRC,' in VANET '04, Philadelphia, PA, USA, Oct. 2004.
[31] M. Torrent-Moreno, D. Jiang, and H. Hartenstein, "Broadcast Reception Rates and Effects of Priority Access in 802.11-based VANETs," in VANET '04, Philadelphia, PA, USA, Oct. 2004.

[32] M. Hazewinkel, Ed., Encyclopaedia of Mathematics. Springer-V., 2002.

[33] W. Feller, An Introduction to Probability Theory and Its Applications, Volume 1. Wiley, 1968.

[34] S. Diwan, B. Dalla Chiara, and F. Deflorio, "Effect of Vehicle to Vehicle and Vehicle to Infrastructure Communication Systems on Transportation Safety," in AATT '06, Chicago, IL, USA, Aug. 2006.

[35] B. Dalla Chiara, F. Deflorio, and S. Diwan, "Communication Among Vehicles and Infrastructures: Evaluation of Outcomes on Road Safety," in ITS '08, Geneva, Switzerland, Jun. 2008.

[36] "Intelligent Transport Systems (ITS), Vehicular Communications (VC), Basic Set of Applications, Definitions," ETSI TR 102638 V1.1, Tech. Rep., Jun. 2009.

[37] S. Biswas, R. Tatchikou, and F. Dion, "Vehicle-to-Vehicle Wireless Communication Protocols for Enhancing Highway Traffic Safety," IEEE Communications Magazine, vol. 44, Jan. 2006.

[38] T. ElBatt, S. K. Goel, G. Holland, H. Krishnan, and J. Parikh, "Cooperative Collision Warning using Dedicated Short Range Wireless Communications,' in VANET '06, Los Angeles, CA, USA, Sept. 2006.

[39] X. Yang, J. Liu, F. Zhao, and N. Vaidya, "A Vehicle-to-Vehicle Communication Protocol for Cooperative Collision Warning," in MobiQuitous '04, Boston, MA, USA, Aug. 2004.

[40] P. Golle, D. Greene, and J. Staddon, "Detecting and correcting malicious data in VANETs," in VANET '04, Philadelphia, PA, USA, October 2004.

[41] J. Camenisch, S. Hohenberger, M. Kohlweiss, A. Lysyanskaya, and M. Meyerovich, "How to Win the Clone Wars: Efficient Periodic nTimes Anonymous Authentication," in CCS, Alexandria, VA, USA, Oct. 2006.

[42] D. Chaum, "Security Without Identification: Transaction Systems to Make Big Brother Obsolete," Comm. of the ACM, vol. 28, no. 10, pp. 1030-1044, 1985.

[43] M. Gerlach, A. Festag, T. Leinmuller, G. Goldacker, and C. Harsch, "Security architecture for vehicular communication," in WIT 2007, Hamburg, Germany, March 2007

[44] M. Raya and J. Hubaux, "Securing Vehicular Ad Hoc Networks," Journal of Computer Security, vol. 15, no. 1, pp. 39 - 68, 2007.

[45] M. El Zarki, S. Mehrotra, G. Tsudik, and N. Venkatasubramanian, "Security Issues in a Future Vehicular Network," in EW '02, Florence, Italy, Feb. 2002.

[46] B. Parno and A.Perrig, "Challenges in Securing Vehicular Networks," in HotNets-IV, College Park, MD, USA, Nov. 2005.

[47] M. Jakobsson, X. Wang, and S. Wetzel, "Stealth Attacks in Vehicular Technologies," in VTC-Fall '04), Los Angeles, CA, USA, Sept. 2004.

[48] J. Blum and A. Eskandarian, "The Threat of Intelligent Collisions," IT Professional, vol. Vol. 6, pp. 24-29, 2004.

[49] A. Aijaz, B. Bochow, F. Dötzer, A. Festag, M. Gerlach, R. Kroh, and T. Leinmüller, "Attacks on Intervehicle Communication Systems - an Analysis," in WIT '06, Hamburg, Germany, Mar. 2006.

[50] P. Papadimitratos, V. Gligor, and J.-P. Hubaux, "Securing Vehicular Communications - Assumptions, Requirements, and Principles," in ESCAR '06, Berlin, Germany, Nov. 2006.

[51] M. Li, R. Poovendran, K. Sampigethaya, and L. Huang, "CARAVAN: Providing Location Privacy for VANET," in ESCAR '05, Cologne, Germany, November 2005

[52] M. Raya, P. Papadimitratos, I. Aad, D. Jungels, and J.-P. Hubaux, "Eviction of Misbehaving and Faulty Nodes in Vehicular Networks," IEEE JSAC, Oct. 2007.

[53] K. Laberteaux and Y.-C.Hu, "Strong VANET Security on a Budget," in ESCAR 06, Berlin, Germany, Nov. 2006.

[54] J. Haas, Y.-C. Hu, and K. Laberteaux, "Real-World VANET Security Protocol Performance," in Globecom, Honolulu, HI, Nov. 2009.

[55] K. Zeng, "Pseudonymous PKI for Ubiquitous Computing," in EuroPKI, Turin, Italy, Jun. 2006.

[56] F. Armknecht, A. Festag, D. Westhoff, and K. Zeng, "Cross-layer Privacy Enhancement and Non-repudiation in Vehicular Communication," in WMAN 07, Bern, Switzerland, Mar. 2007.

[57] F. Kargl, E. Schoch, B. Wiedersheim, and T. Leinmüller, "Secure and Efficient Beaconing for Vehicular Networks," in VANET '08, San Francisco, CA, USA, 2008.

[58] X. Lin, X. Sun, P.-H. Ho, and X. Shen, "GSIS: A Secure and PrivacyPreserving Protocol for Vehicular Communications," IEEE TVT, vol. 56, no. 6, Nov. 2007.

[59] R. Lu, X. Lin, H. Zhu, P.-H. Ho, and X. Shen, "ECPP: Efficient Conditional Privacy Preservation Protocol for Secure Vehicular Communications," in INFOCOM '08, Phoenix, AZ, USA, Apr. 2008. 